\title{
Investigation of deep defects in nanocrystalline-Si/Si interfaces using acoustic spectroscopy
}

\author{
Peter Bury*, Štefan Hardoñ, Hikaru Kobayashi ${ }^{* *}$, Kento Imamura ${ }^{* *}$
}

\begin{abstract}
A set of structures with nanocrystalline-Si/Si interfaces formed on p-type $\mathrm{Si}$ substrate appropriated for photovoltaic application was prepared. The Acoustic DLTS technique based on the acoustoelectric response signal produced by the structure when a longitudinal acoustic wave propagates through the structure was used together with electric characterization to determine deep defects and the role of both individual layers. Several kinds of interface deep centers with activation energies typical for dangling bonds, oxygen participated $\mathrm{Si}$ or point defects were observed as well as a particular influence of individual layers on the interface states. The obtained results are analyzed, discussed and subsequently compared.
\end{abstract}

K e y w or d s: nanocrystalline-Si/Si interfaces, acoustic DLTS, deep defects

\section{Introduction}

Among the various structures and approaches, nanocrystalline silicon (nc-Si) thin films have received great attention for their potential application in various devices, eg solar cells and thin film transistors in liquid crystal displays, compared to amorphous silicon (a-Si) thin films with much less stability. In particular, it is expected that nc-Si can be used to fabricate Si-based optoelectronic devices in near future. It is known that quantum size effects of Si nanocrystallites are the main source for the unique and useful features of the materials and devices, including widening of the optical band-gap which results in visible photoluminescence (PL) [1]. The efficient visible PL at room temperature from nc-Si was the focus of interest because it might lead to the development of a Si-based efficient light source. Due to the excellent quantum confinement effect, nanosized $\mathrm{Si}$ is also advanced material for solar cells, and photonic applications. There, nc-Si as an absorber based tandem solar cell is promising way to exceed the limit of efficiency of single-junction solar cell, since tandem solar cell could cover most solar spectra, compared to single solar structure [2].

Si nanocrystals can be produced usually using two different physical techniques; one is re-crystallization of a-Si films as indirect technique, and the other is direct deposition. The first technique, rapid thermal annealing [3], has difficulties in accurate control of the crystalline volume fraction and grain size. Moreover, post-annealing in an inert atmosphere at high temperatures is generally required for crystallization of Si nanoparticles. Such a high annealing temperature inevitably limits its further applications to optoelectronic devices. On the other hand, a variety of direct chemical vapor deposition (CVD) techniques such as plasma enhanced CVD (PECVD) or low- pressure CVD (LPCVD) have been used to yield materials with good optoelectronic properties $[4,5]$. Though deposition conditions (etching processes, kinetics of the film growth) play an important role in the growth of the nc-Si film, a low processing temperature allows to use of low-cost substrates, which enlarges the application field of nc-Si films in wider industrial production. Therefore, development of a technique to lower the processing temperatures is a challenging task for the semiconductor research community. Recently, a method of formation of ultra-low reflectivity Si surfaces (surface structure chemical transfer (SSCT) method) has been developed [6]. When a Pt mesh is contacted with $\mathrm{Si}$ wafers immersed in $\mathrm{H}_{2} \mathrm{O}_{2}$ plus HF solutions, the mesh structure is instantaneously transferred to the Si surfaces and simultaneously, a nc-Si layer is formed on the surface, resulting in an ultra-low reflectivity. Several models of charge storage were proposed such as three-dimensional quantum confinement effect [7] and interface and defects states [8]. The existence of defects due to lattice damage from CVD process [9] in the nc-Si based MOS structure might generate parasitic traps and influence the charge performance. In this contribution, nc-Si layers deposited by PECVD or SSCT methods on p-type Si substrate to form different structures are investigated by the acoustic DLTS (A-DLTS) technique to characterize the deep centers and the role of individual layers. The obtained results are analyzed, discussed and compared.

\section{Experimental}

The acoustoelectric investigation of deep centers was applied to several structures with nanocrystalline Si prepared on p-type $\mathrm{Si}(100)$ substrates with $10-15 \Omega \mathrm{cm}$ re-

* Department of Physics, Faculty of Electrical Engineering, University of Žilina, Univerzitná 1 , 01026 Žilina, Slovakia, ** Institute of Scientific and Industrial Research, Osaka University, CREST, Japan Science and Technology Organization, 8-1 Mihogaoka, Ibaraki, Osaka 567-0047, Japan, peter.bury@fel.uniza.sk 


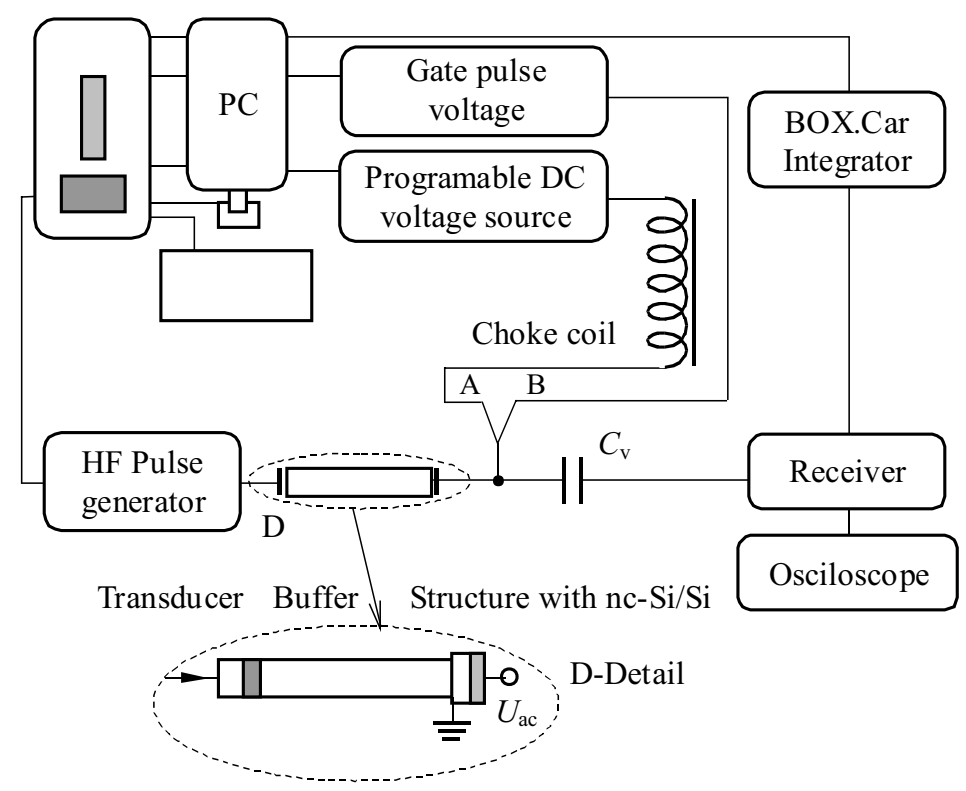

Fig. 1. Block diagram of the experimental setup with the detailed sample configurations (D-detail) for A-DLTS measurement (key in B position)

sistivity: - Nanocrystalline Si (multicolor)/p-Si (100) (No 1-2). The thickness of the nanocrystalline Si layer was 100 - 150nm, surface roughness approx $0.1 \mathrm{~nm}$. Aluminum electrodes were evaporated on both sides. The nanocrystalline Si layer was produced by the PECVD method. Nanocrystalline $\mathrm{Si} / \mathrm{n}-\mathrm{Si} / \mathrm{p}-\mathrm{Si}$ (substrate) /n-Si/ nanocrystalline Si (No 2-1). The nanocrystalline Si layer was produced on the front and rear Si surfaces, followed by phosphorus diffusion on both sides. Aluminum electrodes were evaporated on both sides. $\mathrm{SiO}_{2} /$ nanocrystalline $\mathrm{Si} / \mathrm{p}$-Si (substrate) $/ \mathrm{SiO}_{2}$ (No 2-2). The $\mathrm{SiO}_{2}$ layer was formed by nitric acid oxidation of $\mathrm{Si}$ (NAOS) method, followed by thermal oxidation. The $\mathrm{SiO}_{2}$ thickness is approximately $5 \mathrm{~nm}$. Aluminum electrodes were evaporated on both sides. Ag front electrode/ nanocrystalline $\mathrm{Si} / \mathrm{n}-\mathrm{Si} / \mathrm{p}-$ $\mathrm{Si}$ (substrate)/Al rear electrode (No 2-3). Nanocrystalline $\mathrm{Si}$ layers in this structure was produced by the SSCT method [5], followed by formation of pn-junction. The specimen possesses solar cell structure.

The experimental arrangement of the measurement technique for both measurements of A-DLTS spectra is shown in the block diagram in Fig. 1. The A-B key represents the different arrangement in the case of these individual techniques (A-DLTS in B position). The computer using the LABVIEW system was used to trigger the apparatus Pulse Modulator and Receiver (MATEC 7700), to drive bias voltage as well as to record and evaluate isothermal transients. A longitudinal acoustic wave of frequency $13.2 \mathrm{MHz}$ was generated using $\mathrm{LiNbO}_{3}$ transducer in the arrangement illustrated in the D-detail. The ARS produced by the structure with nc-Si/Si interface was then detected by Receiver, selected using the Gated Integrator and Box-car Averager (SRS), recorded and stored by a computer. The quiescent bias voltage pulses of 100-200 ms with filling traps completely were applied to the structure to measure ARS transients. The differential ARS, $\delta U_{\text {ac }}$ was then monitored as a function of temperature and peaks with maxima of the temperature for which the emission rate is the same as the adjusted sample rate are observed in A-DLTS spectra. The A-DLTS experimental results were at temperatures varying between 373 and $77 \mathrm{~K}$, samples were cooled in nitrogen cryostat.

To know the electrical characteristics, the currentvoltage $(I-V)$ and capacitance voltage $(C-V)$ characteristics were recorded with FLUKE PM 6306 programable automatic RLC meter.

\section{Results and discussion}

The principle of A-DLTS technique [11] is based on the fact that the change of the amplitude of the measured ARS, $\delta U_{\mathrm{ac}}^{0}$ after an injection pulse has been applied is proportional to the nonequilibrium charge at the interface and the decay time constant associated with the relaxation of the ARS amplitude is then a direct measure of the time constant associated with the relaxation processes of injected carriers. Therefore, the ARS amplitude time dependence can be written as

$$
U_{\mathrm{ac}}^{0}(t)=U_{0}+U_{1} \exp \left(-\frac{t}{\tau}\right)
$$

where $U_{0}$ is the original ARS due to the acoustoelectric interaction of acoustic wave and charge at the interface of investigated structure and U1 represents the increase of the ARS due to the injection pulse. The present measurement technique of A-DLTS is based on the computerevaluated transients measured at fixed temperatures. The differential ARS, $\delta U_{\mathrm{ac}}^{0}$, is then monitored as a function of temperature and peaks with maxima at the temperature 


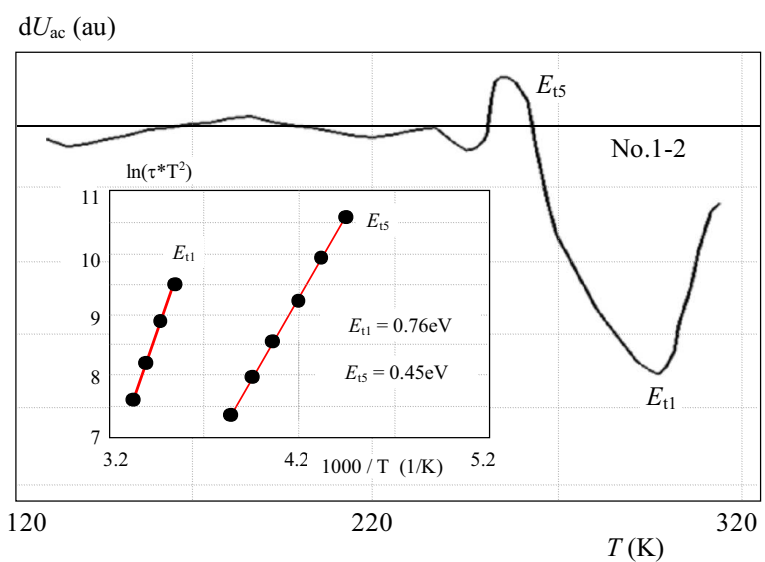

Fig. 2. A-DLTS spectrum of structure No.1-2 and calculated Arrhenius plots (inside)

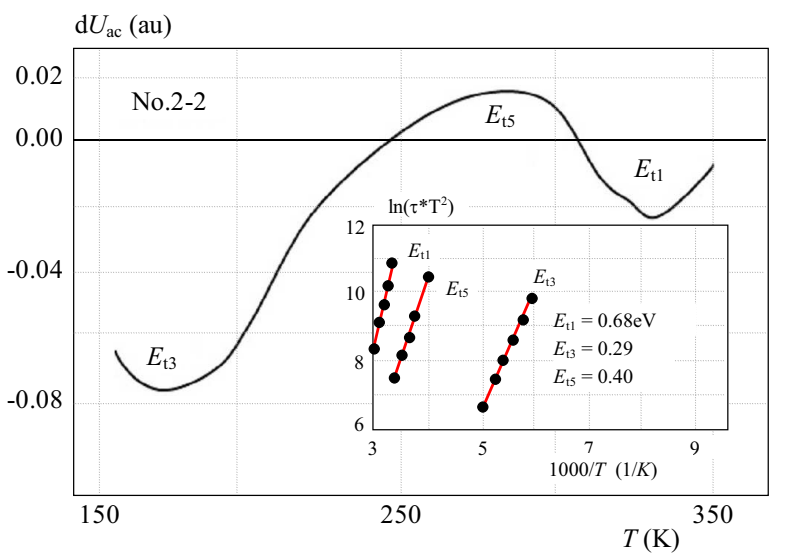

Fig. 4. A-DLTS spectrum of structure No.2-2 and calculated Arrhenius plots (inside)

for which the emission rate is the same as the adjusted sample rate window represent A-DLTS spectra [10, 11, 12].The principle of our A-DLTS measurements consists then in the special analysis of the acoustoelectric transient signal after an injection pulse using a set of emission rate windows similarly as in the case of the DLTS technique developed for the capacitance transients. The software for the calculation allows to use the acoustic version of Lang original scheme or the correlation procedure with higher on/line filters and rectangular weighting function or FTT analysis that can describe more effectively dynamic processes and by that also acoustoelectric responses.

The A-DLTS spectrum of the third structure (No.2-2) is illustrated, together with Arrhenius plots and calculated energies in Fig.4. The presented A-DLTS spectrum that was observed using a pulse voltage $\Delta U_{g}=3.0 \mathrm{~V}$ $\left(U_{g}=-2.0 \mathrm{~V}\right)$ contains three broad peaks, two negative with maxima at $330 \mathrm{~K}$ and $\approx 180 \mathrm{~K}$, respectively and one opposite peak with maximum at $\approx 285 \mathrm{~K}$ and positive double peak with maximum at $281 \mathrm{~K}$ and $265 \mathrm{~K}$, respectively. Another weaker negative peak was detected at a lower temperature $(237 \mathrm{~K})$. The A-DLTS spectrum of the fourth structure, No 2-3, that possesses solar cell

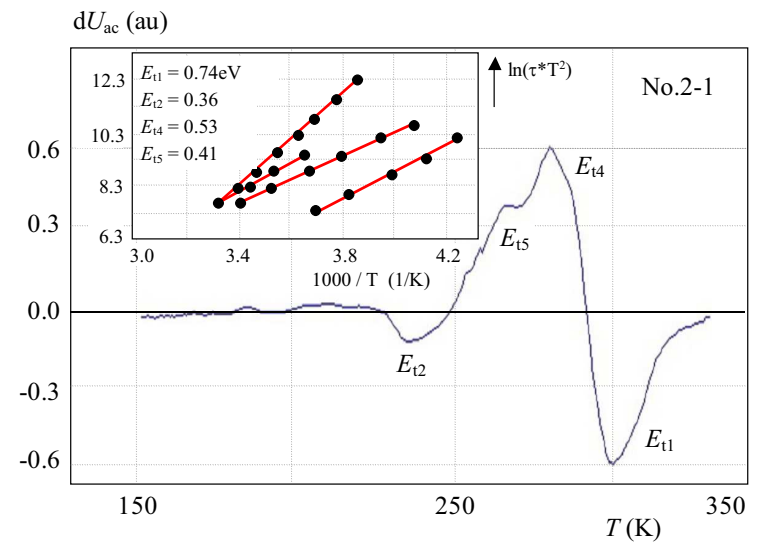

Fig. 3. A-DLTS spectrum of structure No.2-1 and calculated Arrhenius plots (inside)

structure, contains two very weak negative peaks the position of which is similar as in the case of the previous structure.

The summarized set of investigated structures completed about calculated energies and capture cross-sections is in Tab. 1.

The presented parameters of deep centers of investigated set of structures with nc-Si/Si interfaces were obtained using the measured A-DLTS spectra and theoretical principles that were already described in detail [12]. Fig. 2 presents A-DLTS spectrum obtained on the structure No 1-2 prepared on p-type Si with 100-150 nm thick nc-Si and calculated Arrhenius plots including obtained energies, situated inside. The illustrated A-DLTS spectrum that was observed using pulse voltage $\Delta U_{g}=2.0 \mathrm{~V}$ $\left(U_{g}=-1.0 \mathrm{~V}\right)$ contains one evident broad negative peak with maximum at $295 \mathrm{~K}$ with some lateral structure with indication of another peak at lower temperature $(270 \mathrm{~K})$ and much smaller positive peak at $250 \mathrm{~K}$.

Figure 3 presents A-DLTS spectrum obtained on the structure No 2-1 prepared on both sides of p-type Si, front and rear Si surfaces, including calculated Arrhenius plots and obtained energies, inside. The illustrated ADLTS spectrum that was observed using pulse voltage $\left(\Delta U_{g}=2.0 \mathrm{~V}, U_{g}=-1.0 \mathrm{~V}\right)$ contains two quite strong but opposite peaks, negative one with maximum at 300 $\mathrm{K}$ and positive double peak with maxima at $281 \mathrm{~K}$ and $265 \mathrm{~K}$, respectively. Another weaker negative peak was detected at lower temperature $(237 \mathrm{~K})$.

The summarization of all deep centers obtained in investigated structures containing nc-Si/Si interfaces (Tab. 1) indicated the presence of several evident kinds of centers, the most of them are common to several structures. The most frequent deep center is centers with activation energy about $0.68-0.75 \mathrm{eV}$ of acceptor type and centers with activation energies $0.40 \quad 0.45 \mathrm{eV}$ of donor type (No 1-2, No 2-1, No 2-2). In the case of two structures (No 22, No 2-3) deep centers of acceptor type with activation energy $0.25 \mathrm{eV}$ and $0.29 \mathrm{eV}$, respectively, were found as well as deep center with energy $0.53 \mathrm{eV}$ was found in another pair of structures (No 2-1, No 2-3). All these deep 

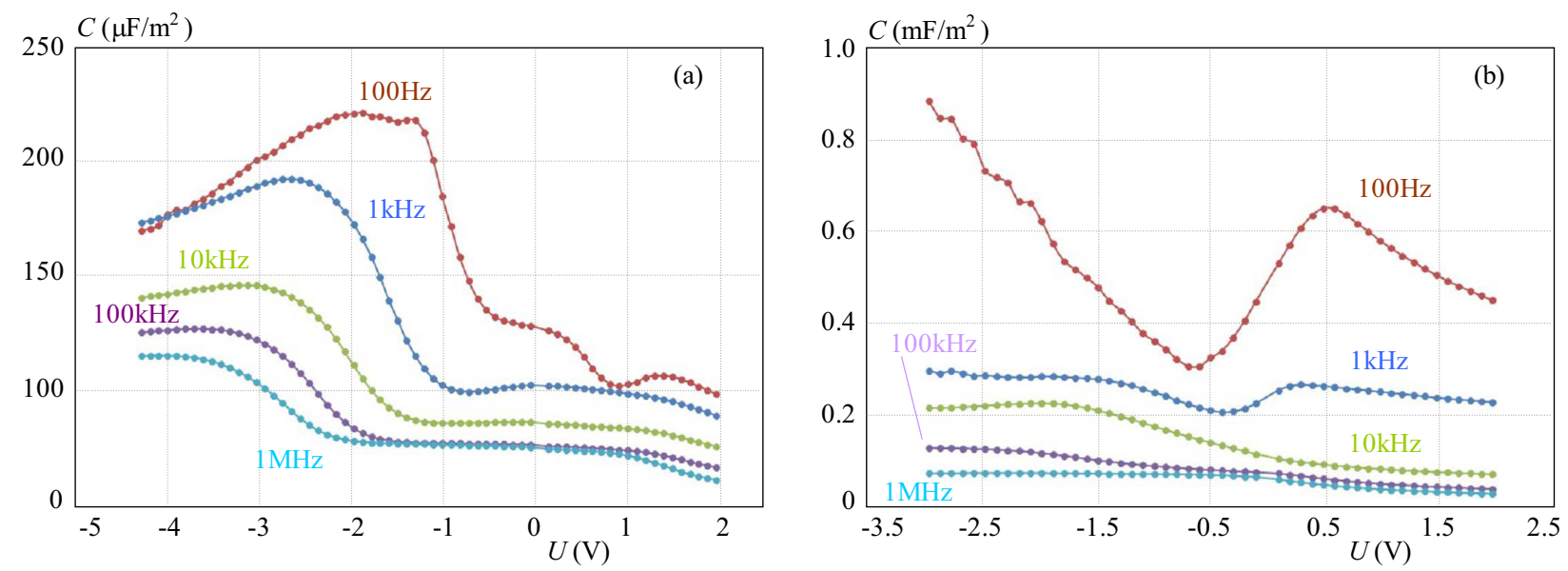

Fig. 5. C-V curves measured at various frequencies of representative structures, (a) - no 1-2 and, (b) no 2-2

Table 1. Summarization of the investigated structures, activation energies and cross-sections provided by the acoustic spectroscopy measurements

\begin{tabular}{lcccc}
\hline Sample & Structure & $E_{\mathrm{t}}(\mathrm{eV})$ & $\sigma\left(\mathrm{cm}^{2}\right)$ & Type \\
\hline No 1-2 & $\mathrm{Al} / \mathrm{Si}(\mathrm{p}) / \mathrm{nc}-\mathrm{Si} / \mathrm{Al}$ & $0.45 \pm 0.05$ & $2.2 \times 10^{-15}$ & $\mathrm{D}$ \\
& & $0.76 \pm 0.05$ & $1.7 \times 10^{-13}$ & $\mathrm{~A}$ \\
\hline No 2-1 & $\mathrm{Al} / \mathrm{nc}-\mathrm{Si} / \mathrm{Si}(\mathrm{n}) / \mathrm{Si}(\mathrm{p})$ & $0.36 \pm 0.02$ & $1.5 \times 10^{-17}$ & $\mathrm{~A}$ \\
& -substrate/Si(n)/nc-Si/Al & $0.41 \pm 0.02$ & $7.3 \times 10^{-18}$ & $\mathrm{D}$ \\
& & $0.53 \pm 0.02$ & $2.1 \times 10^{-15}$ & $\mathrm{D}$ \\
& & $0.74 \pm 0.02$ & $2.3 \times 10^{-13}$ & $\mathrm{~A}$ \\
\hline No 2-2 & $\mathrm{Al} / \mathrm{SiO}_{2} / \mathrm{nc-Si} / \mathrm{Si}(\mathrm{p})$ & $0.29 \pm 0.05$ & $2.3 \times 10^{-16}$ & $\mathrm{~A}$ \\
& (substrate)/Al & $0.42 \pm 0.05$ & $3.7 \times 15^{-18}$ & $\mathrm{D}$ \\
\hline \multirow{2}{*}{ No 2-3 } & $\mathrm{Ag} / \mathrm{nc-Si} / \mathrm{Si}(\mathrm{n}) / \mathrm{Si}(\mathrm{p})$ & $0.25 \pm 0.05$ & $6.5 \times 10^{-14}$ & $\mathrm{~A}$ \\
& (substrate)/Al & $0.53 \pm 0.05$ & $6.3 \times 10^{-15}$ & $\mathrm{~A}$ \\
\hline
\end{tabular}

Note: nc-Si nanocrystalline $\mathrm{Si}, \mathrm{SiO}_{2}$ (NAOS), A acceptor type, D donor type

centers were already detected or assumed in the structure with nc-Si layers.

The origin of deep defects in nc-Si thin films used in photovoltaic application was studied using Raman and photoluminescence spectroscopy subjected to additional investigations [13] and it was found that the defect formation with energy $\approx 0.6 \mathrm{eV}$ resides in the crystalline phase and can be attributed to the dislocation environments in oxygen precipitated c-Si or point defect. The degree of crystallinity itself is responsible for the increase in deep defects creation [14]. The deep defects that act as hole traps with activation energies $0.27 \quad 0.28 \mathrm{eV}$ as well as deep centers with energy close to 0.36 and capture crosssections $10-15$ to $10-16 \mathrm{~cm}^{2}$ were related to impurities in the strain field of extended defects (dislocations or oxygen precipitates) [15].

Concerning the donor types defects, the deep centers with activation energy close to our $0.53 \mathrm{eV}$ was observed in $\mathrm{Si}(\mathrm{n}) / \mathrm{SiO} 2$ with nc-Si nanocrystals inside MOS structure [16] and attributed to quantum levels of the Si quantum dots at around $300 \mathrm{~K}$. However, some other authors [17] suppose that the dangling bonds interacting weakly with oxygen $\left(\mathrm{P}_{\mathrm{bl}}\right)$ are responsible for the deep centers at the nc-Si/Si interfaces that corresponds to our deep centers with activation energies $0.2 \quad 0.3 \mathrm{eV}$ under valence band. Conductivity measurements of the nc-Si films in several configurations confirmed the presence deep defects with energies $0.40 \quad 0.45 \mathrm{eV}[18]$ that are attributed to dangling bonds related to isolated $\mathrm{Si}$ dangling bonds or Si dangling bonds interacting weakly with oxygen or $\mathrm{Si}$ atoms $\left(\mathrm{P}_{\mathrm{bl}}\right)$.

Figure 5 shows $C-V$ curves of representative structures measured at room temperatures and frequencies from $100 \mathrm{~Hz}$ to $1 \mathrm{MHz}$. The humps on the $C-V$ curves that increase at lower frequency imply a high density of localized interface deep centers [19]. However, the comparison of the humps magnitudes for different structures indicates also their specific difference.

\section{Conclusion}

In this study, structures with nc-Si/Si interfaces formed on p-type Si substrate were investigated using ADLTS based on the acoustoelectric response of the inves- 
tigated structures. Several kinds of interface deep defects were obtained, some of them are common to several structures. The acceptor type defects with activation energies in the range $0.250 .29 \mathrm{eV}$ and $\approx 0.7 \mathrm{eV}$ are mostly related to the dislocation environment in oxygen precipitated $\mathrm{Si}$ or point defect. The donor type defects with activation energies $0.45 \quad 0.50 \mathrm{eV}$ or $0.53 \mathrm{eV}$ are attributed mostly to isolated $\mathrm{Si}$ dangling bonds or related to Si dangling bonds interacting weakly with oxygen or silicon atoms. However, the broad peaks of A-DLTS spectra indicated also the broad distribution of interface deep centers in structures with nc-Si/Si interfaces around obtained activation energies.

\section{Acknowledgements}

This work was supported by VEGA project 1/0510/17. This study was also supported by Japan Society for Promotion of Science (JSPS) and Slovak Academy of Science (SAS) under the Japan-Slovakia Research Cooperative Program. The authors would like to thank to Mr F. Cernobila for technical assistance.

\section{REFERENCES}

[1] A. Mossad Ali, H. Kobayashi, T. Inokuma and A. Al-Hajry, Materials Research Bulletin, 48 (2013) 1027.

[2] F. Koch and V. Petrova-Koch, Journal of Non-Crystalline Solids, vol. 198-200, no. 2, ppp. 840-846, 1996.

[3] F. Zhou and J. D. Head, J.Phys.Chem. B 104 (2000) 9981-9986. acoustoelectric response of inves.

[4] A. M. Ali, T. Inokuma, Y. Kurata and S. Hasegawa, Jpn. J. Appl. Phys. 38 (1999) 6047-6053.
[5] A. M. Funde, N. A. Bakr, D. K. Kamble, R. R. Hawaldar, D. P. Amalnerkar and S. R. Jadkar, Sol.Energy Mater. Sol. Cells 92 (2008) 1217-1223.

[6] M. Takahashi, T. Fukushima, Y. Seino, Kim, Woo-Byoung, K. Immamura and H. Kobayashi, Journal of The Electrochemical Society 160 (2013) H443.

[7] M. A. Tischler, R. T. Collins, J. H. Stathis and J. C. Tsang, Applied Physics Letters, vol. 60, no. 5, ppp. 639-641, 1992.

[8] I. M. Chang, S. C. Pan and Y. F. Chen, "”, Physical Review B, vol. 48, no. 12, ppp. 8747, 1993.

[9] K. Dohnalová, L. Ondic, K. Kúsová, I. Pelant, J. L. Rehspringer and R.-R. Mafouana, Journal of Applied Physics, vol. 107, no. 5, ID 053102, 222010.

[10] P. Bury and I. Jamnický, "”, acta phys. slovaca 46, (1996) 693.

[11] P. Bury, T. Matsumoto, I. Bellan, M. Janík and H. Kobayashi, Applied Surface Sciences 269 (2013) 50

[12] P. Bury, I. Bellan, I. Jamnický, J. Kúdelčík and Š. Hardoň, Acta Acustica United with Acustica 102 (2016) 65.

[13] J. D. Fields, B. Gorman, T. Merdzhanova, B. Yan, T. Su and P. C. Taylor, Solar Energy Materials \& Solar Cells 113 (2013) 61.

[14] P. G. Hugger, J. D. Cohen, B. Yan, G. Yue, J. Yang and S. Guha, Appl.Phys.Lett. 97 (2010) 252103.

[15] D. Cavalcoli, A. Cavallini, M. Rosi and S. Pizzini, Fizika i Technika Poluprovodnikov 41 (2007) 435.

[16] T. Lv and L. Zhao, J. Nanomaterials vol. 2014, Article ID 748487.

[17] D. Hiller, M. Ivanescu, A. Stesmans and M. Zacharias, J. Appl. Phys. 107 (2010) 084309

[18] Micard K.Peter, D. Chrastina and G. Isella, http://citeseerx.ist. psu.edu/viewdoc/summary? do=10.1.1.580.3642.

[19] N. Inoue, P. J. Lichtenwalter, J. S. Jur and A. I. Kingon, Jap. J. Appl. Phys. 46, 6480 (2007).

Received 23 April 2017 